\title{
Effekt der Tiefkühllagerung von Wirbelsäulenpräparaten auf deren
}

\section{biomechanisches Verhalten}

Ulrich Schreiber ${ }^{1}$,

Stefan Buchmann ${ }^{1}$,

Erwin Steinhauser ${ }^{1}$,

Wolfram Mittelmeier ${ }^{2}$

Schlagworte:

Wirbelsäule - Biome-

chanik - Lagerung -

Bewegungsumfang -

in vitro
Biomechanische Eigenschaften von biologischen Materialien sind bei deren in-vitro-Prüfung stark vom Prüfaufbau, den Belastungsparametern, aber auch von der Qualität der zu untersuchenden Strukturen abhängig. Da die Versuche meist nicht unmittelbar post mortem durchgeführt werden können, entspricht die Tiefkühllagerung der Präparate bis zur Versuchsdurchführung dem internationalen Standard. In einer Versuchsreihe an bisegmentalen Wirbelsäulenabschnitten aus dem lumbalen Bereich soll gezeigt werden, inwieweit diese Art der Lagerung Veränderungen diskoligamentärer Strukturen und damit des Präparatverhaltens verursacht. Es wurden dazu bovine Wirbelsäulenpräparate im frischen Zustand und nach Tiefkühllagerung in einem Wirbelsäulenprüfstand mit je einem Moment von $\pm 7,5 \mathrm{Nm}$ in den drei Hauptbewegungsebenen belastet. Durch ein berührungsloses, optisches 3-dimensionales Bewegungsanalysesystem wurde die interkorporelle Bewegung ausgewertet. Die Ergebnisse zeigten, dass sowohl in Flexions-/Extensions- als auch in der axialen Rotationsbewegung eine Zunahme des Bewegungsumfangs durch die Lagerung verursacht wurde. In der lateralen Biegung hingegen reduzierte sich der Bewegungsumfang nach dem ersten Lagerungsintervall und stieg dann nach dem zweiten Lagerungsintervall wieder auf fast das im frischen Zustand erreichte Ausmaß an. Es konnte eine sich verändernde Biomechanik der untersuchten Wirbelsäulenabschnitte aufgrund der Tiefkühllagerung festgestellt werden, sodass als Empfehlung für vergleichende in vitro Versuche mit biologischen Material die Testung innerhalb der gleichen Lagerungsintervalle ausgesprochen werden muss.

\section{Einleitung}

Biomechanische in-vitro-Untersuchungen von Wirbelsäulen gehen Fragestellungen der mechanischen Eigenschaften einzelner biologischer Strukturen wie Bänder, Muskeln, Bandscheiben oder den knöchernen Anteilen nach. Verwendung finden dafür sowohl humane Wirbel, als auch wegen der einfacheren Beschaffung, tierische Präparate. Wie Cotterill und Swartz et al. herausfanden, besteht eine gute Vergleichbarkeit der Anatomie und Knochendichte zwischen jungen Kälbern (6-10 Wochen) und humanen Präparaten [1, 2]. Neben den diversen Parametern wie Prüfgeschwindigkeit, Lastrichtung und - größe sind die zu erzielenden Ergebnisse ebenso vom Alter und damit einhergehenden degenerativen Veränderungen der zu untersuchenden Strukturen abhängig. Die Qualität der Präparate hängt aus biomechanischer Sicht außerdem auch von der Art und er Länge der Konservierung $a b$, die zwischen dem Entnahmezeitpunkt und der Testung

${ }^{1}$ Klinik für Orthopädie und Sportorthopädie, Technische Universität M ünchen 2 Orthopädische Klinik und Poliklinik der Universität Rostock vorgenommen werden muss. Als probates Mittel gilt die Tiefkühlkonservierung, die aber auf die einzelnen Strukturen der Wirbelsäule unterschiedliche Auswirkungen hat $[3,4,5$, 6 , 7]. Dynamische Untersuchungen mit Wirbelsäulensegmenten, die sowohl knöcherne Anteile als auch alle disko-ligamentäre Anteile aufweisen, sind indes noch nicht gemacht worden. Mit der hier vorgestellten Versuchsreihe sollte eine mögliche Veränderung des biomechanische Verhaltens von bovinen, lumbalen Wirbelsäulensegmenten durch wiederholte Tiefkühllagerungsintervalle untersucht werden.

\section{Material und Methoden}

Sechs Präparate der Fleckviehrasse mit einem Medianalter von 10 Wochen (Max: 9, Min: 12) wurden für diese Testreihe verwendet. Dabei waren vier der bisegmentalen Präparate der Segmenthöhe L1-L3 und zwei Präparate der Segmenthöhe L4-L6 entnommen (Tab. 1). Um traumatische, degenerative oder metastatisch bedingte Veränderungen auszuschließen, wurden die Präparate vor der Aufnahme in 


\begin{tabular}{|l|c|c|c|c|c|c|}
\hline Präparat & $\mathrm{I}$ & 2 & 3 & 4 & 5 & 6 \\
\hline Geschlecht & - & - & - & - & - & - \\
\hline Alter Wochen] & $\mathrm{I} 0$ & 10 & $\mathrm{I} 2$ & $\mathrm{II}$ & 9 & 9 \\
\hline Segmenthöhe & $\mathrm{LI}-\mathrm{L} 3$ & $\mathrm{~L} 4-\mathrm{L} 6$ & $\mathrm{LI}-\mathrm{L} 3$ & $\mathrm{LI}-\mathrm{L} 3$ & $\mathrm{LI}-\mathrm{L} 3$ & $\mathrm{~L} 4-\mathrm{L} 6$ \\
\hline
\end{tabular}

Tab. I: $\quad$ Liste der bovinen Präparate

die Versuchsreihe makroskopisch und radiologisch untersucht.

Abbildung 1 stellt den Temperaturverlauf der Präparate vom Erhalt, während der einzelnen Lagerungsintervalle (II, V) und Testungen (I, IV, VII) dar. Die Präparate wurden nach Erhalt im frischen Zustand zunächst von allen Weichteilen freipräpariert, dabei wurde insbesondere auf den Erhalt disko-ligamentärer Strukturen geachtet.

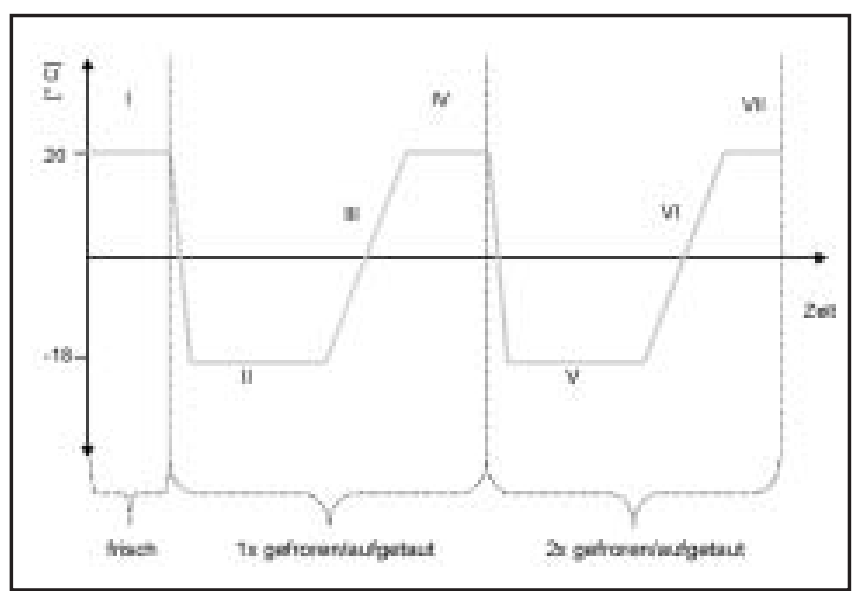

Abb. I: Schematische Darstellung des Temperaturverlaufs der Einfrier/Auftauintervalle

Um eine Standardisierung der Fixierung in dem Wirbelsäulenprüfstand zu gewährleisten wurden, die Präparate in Epoxidharz (Ureol 5202-1A/B, Fa. Ciba, Frankreich) derart eingebettet, dass die kranialen und kaudalen Endplattenmittelpunkte durch die senkrechte Rotationsachse des Aufbaus verlief. Der Mittelpunkt einer Wirbelkörperendplatte ist dabei durch den Schnittpunkt des maximalen sagittalen und transversalen Durchmessers definiert $[8,9,10]$. Die räumliche Ausrichtung der humanen Wirbel wurde von Bernhardt

et al. in vivo radiologisch ermittelt und wurde in dieser Versuchsreihe mit $1^{\circ}$ Kyphose der kranialen Deckplatte eingestellt [11].

Die erste Einfrierdauer (II), nachdem die Präparate frisch getestet (I) wurden, betrug im Median 13 Tage und die Lagerung (V) bis zum dritten Versuchsschritt dauerte im Median 12 Tage. Für die Lagerung wurden die Präparate in abgedichteten Gefrierbeuteln bei $-18^{\circ} \mathrm{C}$ tiefgefroren. Das Auftauen der Präparate erfolgte über ca. 10 Stunden im Kühlschrank bei $4^{\circ} \mathrm{C}$ (III und VI).

Die biomechanische Testung erfolgte in einem Wirbelsäulenprüfstand [12] (Abb. 2). Die gewählte, alternierende Belastung in allen drei Hauptbewegungsebenen (Sagittal-, Frontal- und Transversalebene) von $\pm 7,5 \mathrm{Nm}$ entspricht der in den Richtlinien der Deutschen Gesellschaft für Wirbelsäulenchirurgie vorgegebenen Belastung für den thorakolumbalen Bereich [13].

Es kann davon ausgegangen werden, dass die biologischen Strukturen bei der applizierten Belastungshöhe nicht irreversibel beansprucht werden und somit fortlaufende Testreihen an ein und demselben Präparat gerechtfertig sind. ImGegensatz zu den Belastungen in der Frontal- und Sagittalebene mit einer exzentrischen Krafteinleitung $( \pm 20,0 \mathrm{~N}$, Hebelarm: $37,5 \mathrm{~cm}$ ) erfolgte die Rotation in der Transversalebene mit Hilfe eines reinen Momentes (Abb. 2).

Die Datenaufzeichnung und -auswertung der einzelnen Segmentbewegungen erfolgte mit einem berührungslosen, optischen Messsystem (SimiMotion, Version 5.2, SIMI Reality Motion Systems). Dazu wurden die Wirbel jeweils mit drei Pinmarkern versehen, die während der Testung durch zwei Digitalkameras (DCR-TRV900E, Sony) aufgezeichnet wurden. Die Messsignale der Kraft- (200 N, Typ 8435-5200; Burster) und Momentensensoren (100 Nm, Typ 8628-5100; Burster) wurden mit Hilfe einer Datenerfassungssoftware (LabView 5.0, National Instruments) aufgezeichnet. Ausgewertet wurde jeweils der dritte Belastungszyklus, um Anlauf- und Setzeffekte innerhalb der Präparate und des Prüfstandes ausschließen zu können. Ausgewertet wurden die einzelnen maximalen Bewegungsumfänge (RoM) bei $\pm 7,5 \mathrm{Nm}$ und die neutralen Zonen (NZ) bei $\pm 0,0 \mathrm{Nm}$ der Präparate.

\section{Ergebnisse}

Die Tiefkühllagerung beeinflusste das Ausmaß der maximalen Bewegungen und der neutralen Zone in allen Bewegungsebenen. So ergaben sich in der Flexionsals auch in der Extensionsbewegung sowohl für die neutrale Zone als auch für die maximale Bewegung größere Umfänge bei der Testung nach einmaligen und zweimaligen Lagerungsintervall gegenüber den im frischen Zustand ermittelten Werten (Abb. 3).

Im frischen Zustand wurde eine Gesamt-RoM von $8,7^{\circ}$ (Mittelwert) $\pm 0,4^{\circ}$ (Standardabweichung) und eine Gesamt- NZ von $5,3^{\circ} \pm 0,6^{\circ}$ ermittelt. Die Testung nach dem ersten Lagerungsintervall ergab $9,1^{\circ} \pm 1,1^{\circ}$ Gesamt-RoM und $5,7^{\circ} \pm 1,3^{\circ}$ Gesamt-NZ. Eine weitere Steigerung war ergab die Messung nach dem zweiten Lagerungsintervall mit $10,7^{\circ} \pm 1,0^{\circ}$ GesamtRoM und $6,4^{\circ} \pm 1,0^{\circ}$ Gesamt- $N Z$.

Auch in der Transversalebene war ein qualita-

Abb. 2: Funktionsskizze des Wirbelsäulenprüfstandes
Transiationi ISctwerkan: (Traral. + Ret) iFotation! $37.5 \mathrm{tm}$ samt-RoM erhöhte sich hier von $11,4^{\circ} \pm 1,3^{\circ}$ 
ORIGINAL ARBEITEN

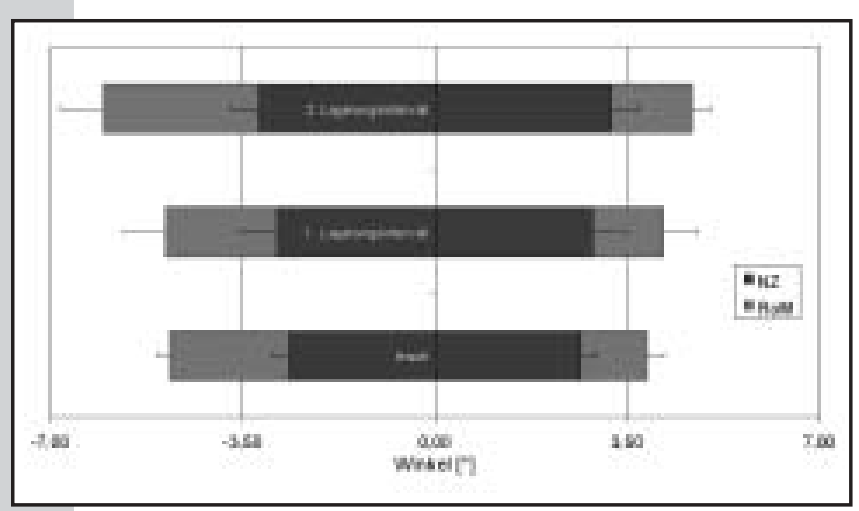

Abb. 3: Maximaler Bewegungsumfang und neutrale Zone in Flexions-Extensionsbewegung mit Standardabweichungen

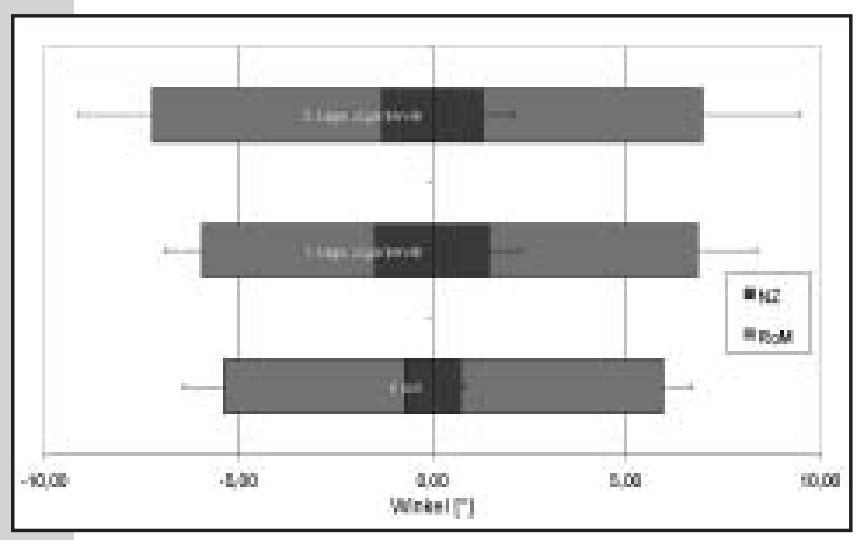

Abb. 4: Maximaler Bewegungsumfang und neutrale Zone in Rotation im und gegen Uhrzeigersinn

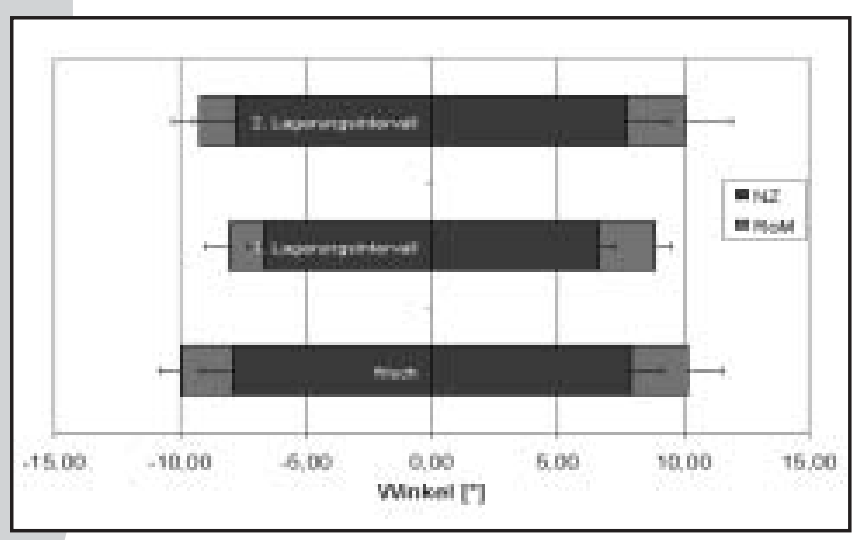

Abb. 5: Maximaler Bewegungsumfang und neutrale Zone in Seitneigung links/rechts

auf $12,8^{\circ} \pm 2,3^{\circ}$ nach dem ersten Lagerungsintervall und nach dem zweiten Lagerungsintervall auf $14,2^{\circ}\left( \pm 5,4^{\circ}\right.$. Die neutrale Zone vergrößerte sich von $1,4^{\circ} \pm 0,3^{\circ}$ auf $2,9^{\circ}$ $\pm 1,6^{\circ}$, reduzierte sich dann aber nach dem zweiten Lagerungsintervall auf $2,6^{\circ}\left( \pm 1,6^{\circ}\right.$ (Abb. 4).

Sowohl die Gesamtauslenkung in der neutralen Zone als auch die Gesamt-RoM wiesen im frischen Präparatszustand in der Seitneigung links/rechts mit $20,1^{\circ} \pm 2,2^{\circ}$ respektive $15,8^{\circ} \pm 2,5^{\circ}$ Maximalwerte auf. Nach dem ersten Lagerungsintervall reduzierten sich diese Werte auf $16,9^{\circ} \pm 1,5^{\circ}$ RoM und $13,3^{\circ} \pm 1,2^{\circ} \mathrm{NZ}$ und erhöhten sich nach dem zweiten Lagerungsintervall auf $19,3^{\circ} \pm 2,9^{\circ}$ und $15,6^{\circ} \pm 3,4^{\circ}$ (Abb. 5).
Effekt der Tiefkühllagerung von Wirbelsäulenpräparaten auf deren biomechanisches Verhalten

\section{Diskussion}

Wie Untersuchungen der mechanischen Eigenschaft knöcherner Strukturen mit Schraubenausrissversuchen über einen längeren Zeitraum (3-5 Tage) gezeigt haben, hat der autolytische Prozess keinen signifikanten Einfluss auf das Ausrissverhalten [14]. Dhillon et al. und Gleizes et al. konnten bei Kriechversuchen mit tiefkühl-konservierten Bandscheiben ebenso keine Veränderungen der viskoelastischen Eigenschaften feststellen [3; 15]. Zu dazu konträren Ergebnissen kamen jedoch Bass et al., Clavert et al. und Van Ee et al., die erhebliche veränderte biomechanische Antworten auf Untersuchungen an Schweinebandscheiben, humanen Sehnen und Kaninchenmuskeln festgestellt hatten [4, 16, 17]. In der vorliegenden Studie sollte das Zusammenspiel der funktionellen Einheiten der Wirbel mit intakten disko-ligamentären Strukturen durch die Verwendung bisegmentaler, boviner Wirbelsäulensegmente sowohl im frischen als auch nach Intervallen der Tiefkühllagerung untersucht werden und damit im Gegensatz zu den oben beschriebenen, reduzierten Versuchen stehen. Prozentual lagen die Änderungen zu der im frischen Zustand erreichten RoM je nach Belastungsebene bei einer Bewegungszunahme von bis zu $25 \%$ und einer Reduzierung um $16 \%$. Für die neutrale Zone stellte sich sogar eine noch größere Bandbreite ein. Der Unterschied betrug hier zwischen einer Vergrößerung um 104,8\% und einer Verminderung um 16,0\%. Diese Veränderungen des biomechanischen Verhaltens können durch das Austrocknen der disko-ligamentären Strukturen erklärt werden. Sowohl die Tiefkühllagerung als auch die Umgebungsbedingungen (Standard-Laborbedingungen) verursachen ein Verspröden dieser Strukturen. Durch die Belastung werden die spröden Fasern geschädigt, was konsekutiv zu einer Vergrößerung der Bewegungsumfänge führt. Aufgrund der Muskel-/Faserstrukturen zwischen den Querfortsätzen, der großen Ansatzfläche und des großen Hebelarmes zur Längsachse des Wirbels, kam es erst nach dem zweiten Lagerungsintervall, in der Frontalebene ebenfalls zur Schädigung der Fasern.

Diese Ergebnisse der sich durch die Tiefkühllagerung stark ändernden Biomechanik, lassen den Schluss zu, dass vergleichende Untersuchungen nur mit gleicher Lagerungshistorie zulässig sind. Durch die kostengünstige und einfache Art ist die Tiefkühlkonservierung jedoch immer noch als probat anzusehen.

\section{Abstract}

The biomechanical behaviour of biological material while tested in vitro strongly depends on the setup, the parameters concerning the force application and also on the quality of the investigated structures. Due to the fact that tests are usually not carried out immediately post mortem, deep-freeze-storage of the specimens is the golden standard. The hereafter described test sequences investigated what influence this kind of storage has on the disco-ligamental structures of bovine, bisegmental spinal specimens. Six lumbar specimens where tested in a spinal test jig both fresh and after deep-freeze-storage. A load of maximum (7,5 Nm was applied in all of the three anatomical planes. Motion analysis was performed by a contactless three dimensional optical measuring system. As a result flexion/extension and axial rotation showed an increase of range of motion due to the storage. In lateral bending in contrary after the first storage interval a reduction in the range of motion was investigated followed by an increase after the second storage interval. A change in the biomechanical behaviour of the tested spinal specimens was detected due to deep-freeze-storage. The- 


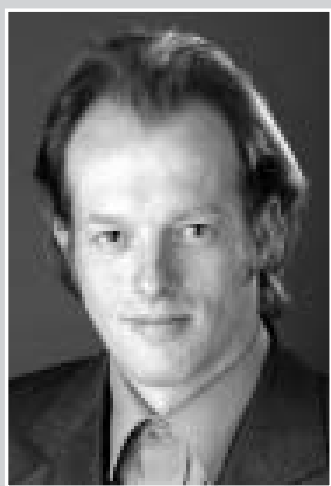

\section{Dipl.-Ing. Ulrich Schreiber}

Klinik für Orthopädie und Sportorthopädie Abteilung Biomechanik Technische Universität München

Connollystr. 32

D-80809 München

Tel.: 089/ 28924490

Fax: 089/28924494

e-mail:

u.schreiber@Irz.tum.de

Akademischer Lebenslauf:

1988-94 Studium, Allgemeiner Maschinenbau an der Technischen Universität München

1995 Diplomarbeit am Lehrstuhl für Fügetechnik der Technischen Universität München: Entwicklung und Konstruktion einer dynamischen Prüfmaschine für Dauerschwingversuche nach DIN 53285

seit 1997 Wissenschaftlicher Mitarbeiter an der Klinik für Orthopädie und Sportorthopädie, Abteilung Biomechanik der Technischen Universität München (Direktor: Prof. Dr. R. Gradinger)

refore as a recommendation in case of a comparing in vitro investigation with biological material involved, tests have to be performed within the same storage interval.

\section{Literatur}

[1] Cotterill P. C., Kostuik J. P., D'Angelo G., Fernie G. R., Maki B. E.: An anatomical comparison of the human and bovine thoracolumbar spine. J.Orthop.Res. 4, 298-303, 1986

[2] Swartz D. E., Wittenberg R. H., Shea M., White A. A., III, Hayes W. C.: Physical and mechanical properties of calf lumbosacral trabecular bone. J.Biomech. 24, 1059-1068, 1991

[3] Dhillon N., Bass E. C., Lotz J. C.: Effect of frozen storage on the creep behavior of human intervertebral discs. Spine $26,883-888,2001$

[4] Bass E. C., Duncan N. A., Hariharan J. S., Dusick J., Bueff H. U., Lotz,J. C.: Frozen storage affects the compressive creep behavior of the porcine intervertebral disc. Spine 22, 2867-2876, 1997

[5] Clavert P., Kempf J . F., Bonnomet F., Boutemy P., Marcelin L., Kahn,J. L.: Effects of freezing/thawing on the biomechanical properties of human tendons. Surg.Radiol.Anat. 23, 259-262, 2001a

[6] Van Ee C. A., Chasse A. L., Myers B. S.: Quantifying skeletal muscle properties in cadaveric test specimens: effects of mechanical loading, postmortem time, and freezer storage. J Biomech.Eng 122, 9-14, 2000a

[7] Gleizes V., Viguier E., Feron J. M., Canivet S., Lavaste F.: Effects of freezing on the biomechanics of the intervertebral disc. Surg.Radiol.Anat. 20, 403-407, 1998a
[8] Berry J. L., Moran J. M., Berg W. S., and Steffee A. D.: A morphometric study of human lumbar and selected thoracic vertebrae. Spine 12, 362-367, 1987

[9] Hall L. T., Esses S .I., Noble P. C., and Kamaric E.: Morphology of the lumbar vertebral endplates. Spine 23, 15171522, 1998

[10] Panjabi M. M., Goel V., Oxland T., Takata K., Duranceau J., Krag M., Price M.: Human lumbar vertebrae. Quantitative three-dimensional anatomy. Spine 17, 299-306, 1992

[11] Bernhardt M. Bridwell K. H.: Segmental analysis of the sagittal plane alignment of the normal thoracic and lumbar spines and thoracolumbar junction. Spine 14, 717-721, 1989

[12] Schreiber U., Bence T., Baader R., Beisse R., Grupp T., Mittelmeier W., Steinhauser E.: Validation of a newly developed experimental setup for segmental spinal testing. Eur.Spine J. 34, S65-66, 2001

[13] Wilke H. J., Wenger K., Claes L.: Testing criteria for spinal implants: recommendations for the standardization of in vitro stability testing of spinal implants. Eur.Spine J. 7, 148154, 1998

[14] Kaab M. J., Putz R., Gebauer D., Plitz W.: Changes in cadaveric cancellous vertebral bone strength in relation to time. A biomechanical investigation. Spine 23, 1215-1219, 1998

[15] Gleizes V., Viguier E., Feron J. M., Canivet S., Lavaste $F .:$ Effects of freezing on the biomechanics of the intervertebral disc. Surg.Radiol.Anat. 20, 403-407, 1998b

[16] Clavert P., Kempf J. F., Bonnomet F., Boutemy P., Marcelin L., Kahn J. L.: Effects of freezing/thawing on the biomechanical properties of human tendons. Surg.Radiol.Anat. 23, 259-262, 2001b

[17] Van Ee C. A., Chasse A. L., Myers B. S.: Quantifying skeletal muscle properties in cadaveric test specimens: effects of mechanical loading, postmortem time, and freezer storage. J Biomech.Eng 122, 9-14, 200b 